\title{
The significance of diagnostic delay in endometriosis
}

\section{Editorial}

Endometriosis is a common gynecological disorder, which affects $5-15 \%$ of women of reproductive age, with a varied prevalence ${ }^{1}$ depending on the population in question and the presence of infertility ${ }^{2}$ and an unpredictable rate of progression. ${ }^{3}$ Endometriosis has a chronic nature which often leads to deterioration in quality of life and high psychological morbidity. ${ }^{4,5}$ Epidemiological studies highlight a high prevalence of chronic pelvic pain in community care settings, with almost half of these women diagnosed as having endometriosis. ${ }^{6}$

The gold standard for diagnosis of endometriosis is surgery, which is advocated as a second line investigation after failure of therapeutic intervention for the management of chronic pelvic pain. ${ }^{7}$ Despite progress, the diagnosis of endometriosis remains a clinical challenge with many patients left undiagnosed with an estimated average delay of up to 10.4years. ${ }^{5,8-10}$ Two thirds of women with endometriosis are initially misdiagnosed and almost half are examined by five physicians or more before a correct diagnosis is made. ${ }^{11}$ Diagnostic delay is significantly longer in women presenting with pelvic pain in comparison to those presenting with infertility. ${ }^{5,12,13}$ In addition, delay before surgical diagnosis of deep infiltrating endometriosis is significantly longer for patients with advanced stage IV disease than for those with stage I, II or III disease. ${ }^{14}$ The endometriosis-associated costs to society are aggravated by delayed diagnosis and empirical treatments, as are the costs to the individual when disease symptoms interfere with daily function. ${ }^{5,15,16}$

Diagnostic delay in endometriosis is normally considered as the time interval between the appearance of symptoms and the performance of diagnostic surgery. Recently, Nnoaham et al. ${ }^{5}$ described a delay of 6.7 years in affected women, which was mainly due to delays in referral from the primary care physician to the gynecologist, with women reporting an average of seven visits before specialist referral. Ballard et al. ${ }^{17}$ investigated possible reasons for the diagnostic delay in endometriosis using a qualitative questionnaire given to women attending a pelvic pain clinic. ${ }^{17}$ The authors found that delays in the diagnosis of endometriosis occur at both an individual patient level and at a medical healthcare system level. At an individual level; women bore symptoms due to inaccurate perception of normal versus abnormal pain, embarrassment, endurance and individual coping strategies; while general practitioners and family doctors tend to normalize symptoms, symptoms are intermittently suppressed through hormones and nondiscriminatory investigations such as a normal transvaginal scan are relied upon. They highlighted the importance of an early diagnosis for women who suffer at physical, emotional, and social levels when they remain undiagnosed. Other possible reasons for this delay may be related to a lack of awareness or knowledge, or simply lack of confidence in surgery results. Early diagnosis of endometriosis refers, by definition, to early surgery, since surgery is the gold standard for diagnosis. But early surgery is not advocated for all patients. In certain cases, empirical treatment is strongly recommended.

Laparoscopic surgery under general anesthesia is most commonly required to reach a definitive diagnosis of endometriosis, but this is expensive and potentially associated with complications. ${ }^{18}$
Volume 2 Issue I - 2016

\author{
Adi YWeintraub \\ Department of Obstetrics and Gynecology, Soroka University \\ Medical Center, Israel
}

Correspondence: Adi Y Weintraub, Department of Obstetrics and Gynecology, Soroka University Medical Center, Faculty of Health Sciences, Ben-Gurion University of the Negev, Beer Sheva, Israel, Tel 054-77I-7053, Email adiyehud@bgu.ac.il

Received: January 26, 2016 | Published: February 03, 2016

Numerous reasons have been advocated in an attempt to explain the diagnostic delay of endometriosis. Traditionally these could be divided into three groups:

i. Disease related factors, such as overlapping of endometriosis symptoms with other morbidities (i.e., urinary tract infection, interstitial cystitis, pelvic inflammatory disease and others), and the lack of good nonsurgical methods of diagnosing the disease or at least predicting its presence.

ii. Patient related factors, including symptom endurance due to inaccurate perception of normal versus abnormal pain, embarrassment, and individual coping strategies.

iii. Physician related factors, such as lack of awareness or knowledge, or simply lack of confidence in surgery results.

Although these traditional explanations for the delay in diagnosis may account for a large portion of the delays, I would like to point out some additional factors that may be even more substantial. These have to do with health care medical policy. There is a considerable void in clinical guidelines to direct clinicians regarding the appropriate investigation and appropriate modality, timing and provision of adequate treatment. These could be explained by several factors including:

i. A diversity in symptoms (cysts, pain, infertility or a combination of these) and in clinical settings (adolescence, chronic pain patients, patients desiring fertility, etc.), has led to lack of guidelines.

ii. Many of the existing recommendations advocate delaying surgery resulting in a delay in diagnosis.

iii. Due to a shortage in adequately powered randomized controlled trials that aim to answer some of the important clinical questions, good evidence-based recommendations cannot be made.

iv. Some of the existing clinical recommendations are too general at best and contradicting and confusing at times (i.e. Does surgery for ovarian endometrioma improve fertility performance or impair ovarian reserve and which type of patient should it be recommended for?).

In conclusion, many physicians in community practice are still largely unaware of the role of specialized care in the optimal 
management of endometriosis. It is important to dedicate efforts and resources to research and perform randomized clinical trials that will aid in establishing significant evidence based guidelines in an attempt to minimize the delay in diagnosis of endometriosis.

\section{Acknowledgements}

None.

\section{Conflict of interest}

The author declares no conflict of interest.

\section{References}

1. Bulun SE. Endometriosis. N Engl J Med. 2009;360(3):268-279.

2. Olive DL, Schwartz LB. Endometriosis. $N$ Engl $J$ Med. 1993;328(24):1759-1769.

3. Cramer DW. Epidemiology of endometriosis. In: Wilson EA editor. Endometriosis. New York, USA: Alan R Liss publishers; 1985. 5 p.

4. Strathy JH, Molgaard CA, Coulam CB, et al. Endometriosis and infertility: laparoscopic study of endometriosis among fertile and infertile women. Fertil Steril. 1982;38(6):667-672.

5. Eskenazi B, Warner ML. Epidemiology of endometriosis. Obstet Gynecol Clin North Am. 1992;24(2):235-258.

6. Missmer SA, Cramer DW. The epidemiology of endometriosis. Obstet Gynecol Clin North Am. 2003;30(1):1-19.

7. Rogers PA, D Hooghe TM, Fazleabas A, et al. Priorities for endometriosis research: recommendations from an international consensus workshop. Reprod Sci. 2009;16(4):335-346.

8. D Hooghe TM, Hill JA. Endometriosis. In: Berek JS editor. Novak's Gynecology. 14th ed. USA: Lippincott Company; 2006.
9. Sinaii N, Cleary SD, Ballweg ML, et al. High rates of autoimmune and endocrine disorders, fibromyalgia, chronic fatigue syndrome and atopic diseases among women with endometriosis: a survey analysis. Hum Reprod. 2002;17(10):2715-2724.

10. Nnoaham KE, Hummelshoj L, Webster P, et al. Impact of endometriosis on quality of life and work productivity: a multicenter study across ten countries. Fertil Steril. 2011;96(2):366-373.

11. Viganò $\mathrm{P}$, Parazzini F, Somigliana E, et al. Endometriosis and aetiological factors. Best Pract Res Clin Obstet Gynaecol. 2004;18(2):177-200.

12. Royal College of Obstetricians and Gynaecologists. The initial management of chronic pelvic pain. Guideline number 41, London, UK: RCOG; 2005. p. 1-12.

13. Hadfield R, Mardon H, Barlow D, et al. Delay in the diagnosis of endometriosis: a survey of women from the USA and the UK. Hum Reprod. 1996;11(4):878-880

14. Hudelist G, Fritzer N, Thomas A, et al. Diagnostic delay for endometriosis in Austria and Germany: causes and possible consequences. Hum Reprod. 2012;27(12):3412-3416.

15. Husby GK, Haugen RS, Moen MH. Diagnostic delay in women with pain and endometriosis. Acta Obstet Gynecol Scand. 2003;82(7):649653.

16. Hummelshoj L, Prentice A, Groothuis P. Update on endometriosis. Womens Health (Lond Engl). 2006;2(1):53-56

17. Dmowski WP, Lesniewicz R, Rana N, et al. Changing trends in the diagnosis of endometriosis: a comparative study of women with pelvic endometriosis presenting with chronic pelvic pain or infertility. Fertil Steril. 1997;67(2):238-243.

18. Vercellini P, Somigliana E, Viganò $\mathrm{P}$, et al. Surgery for endometriosis-associated infertility: a pragmatic approach. Hum Reprod. 2009;24(2):254 269. 